"Mircea cel Batran" Naval Academy Scientific Bulletin, Volume XX - 2017 - Issue 1 The journal is indexed in: PROQUEST / DOAJ / Crossref / EBSCOhost / INDEX COPERNICUS / DRJI / OAJI I JOURNAL INDEX I I2OR / SCIENCE LIBRARY INDEX / Google Scholar / Academic Keys/ ROAD Open Access I Academic Resources / Scientific Indexing Services / SCIPIO / JIFACTOR

\title{
MESHING AND 3D MODELLING FOR SHIP CONSTRUCTION ELEMENTS
}

\author{
Adrian POPA ${ }^{1}$ \\ Ionut-Cristian SCURTU \\ Beazit ALI ${ }^{3}$ \\ George NOVAC ${ }^{4}$ \\ ${ }^{1}$ Associate Professor PhD. Eng. "Mircea cel Batran" Naval Academy, Constanta, Romania, \\ adrian.popa@anmb.ro \\ ${ }^{2}$ Principal Instructor PhD. Eng."Mircea cel Batran" Naval Academy, Constanta, Romania, \\ ionut.scurtu@anmb.ro \\ ${ }^{3}$ PhD. Eng"Mircea cel Batran" Naval Academy, Constanta, Romania, beazit.ali@anmb.ro \\ ${ }^{4}$ Associate Professor, PhD. Eng"Mircea cel Batran" Naval Academy, Constanta, Romania, \\ george.novac@anmb.ro
}

Abstract: All construction elements are subjected to immense forces during ship operation. The presented study is made using different mesh for high stress area on the transverse element presented. The paper presents static loading analysis for the transverse beam model based on Ansys software results.

Key words: transverse beam, statical loads, ansys software, meshing.

\section{Introduction}

In this paper we analyzed the mechanical resistance of a tranverse beam structure used in naval construction. The content has exposed particularities of the model structure, but operations were performed and numerical computation of Ansys Workbench software. Naval technology development has reached a very advanced nowadays and their development will continue for the purposes of weight reductions, which will have effects even of its design or working parameters. Based on ship loads on calm sea we conducted a series of simulations in order to determine which efforts and mesh is showing the best results. The paper updated the mechanical problem of the items ship static approach using modern software programs specially designed for field engineers.

Units

TABLE 1

\begin{tabular}{|r|c|}
\hline Unit System & $\begin{array}{r}\text { Metric }(\mathrm{m}, \mathrm{kg}, \mathrm{N}, \mathrm{s}, \mathrm{V}, \mathrm{A}) \\
\text { Degrees rad/s Celsius }\end{array}$ \\
\hline Angle & Degrees \\
\hline $\begin{array}{r}\text { Rotational } \\
\text { Velocity }\end{array}$ & $\mathrm{rad} / \mathrm{s}$ \\
\hline Temperature & Celsius \\
\hline
\end{tabular}

Model (A4)

Geometry

TABLE 2

Model (A4) > Geometry

\begin{tabular}{|r|r|}
\hline $\begin{array}{r}\text { Object } \\
\text { Name }\end{array}$ & Geometry \\
\hline State & Fully Defined \\
\hline & Definition \\
\hline
\end{tabular}

\begin{tabular}{|c|c|}
\hline Source & K:I21_filesldp0ISYSIDMISYS.agdb \\
\hline Type & DesignModeler \\
\hline Length Unit & Meters \\
\hline $\begin{array}{r}\text { Element } \\
\text { Control }\end{array}$ & Program Controlled \\
\hline Display Style & Body Color \\
\hline & Bounding Box \\
\hline Length $X$ & $42.017 \mathrm{~m}$ \\
\hline Length $\mathrm{Y}$ & $9.6403 \mathrm{~m}$ \\
\hline Length Z & 10. $\mathrm{m}$ \\
\hline & Properties \\
\hline Volume & $290.41 \mathrm{~m}^{3}$ \\
\hline Mass & $2.2798 \mathrm{e}+006 \mathrm{~kg}$ \\
\hline $\begin{array}{r}\text { Scale Factor } \\
\text { Value }\end{array}$ & 1. \\
\hline & Statistics \\
\hline Bodies & 1 \\
\hline $\begin{array}{r}\text { Active } \\
\text { Bodies }\end{array}$ & 1 \\
\hline Nodes & 48248 \\
\hline Elements & 26366 \\
\hline Mesh Metric & None \\
\hline \multicolumn{2}{|r|}{ Basic Geometry Options } \\
\hline Parameters & Yes \\
\hline $\begin{array}{r}\text { Parameter } \\
\text { Key }\end{array}$ & DS \\
\hline Attributes & No \\
\hline $\begin{array}{r}\text { Named } \\
\text { Selections }\end{array}$ & No \\
\hline Material & No \\
\hline
\end{tabular}


“Mircea cel Batran" Naval Academy Scientific Bulletin, Volume XX - 2017 - Issue 1

The journal is indexed in: PROQUEST / DOAJ / Crossref / EBSCOhost / INDEX COPERNICUS / DRJI / OAJI I JOURNAL INDEX / I2OR / SCIENCE LIBRARY INDEX / Google Scholar / Academic Keys/ ROAD Open Access I Academic Resources / Scientific Indexing Services / SCIPIO / JIFACTOR

\begin{tabular}{|c|c|}
\hline Properties & \\
\hline \multicolumn{2}{|c|}{ Advanced Geometry Options } \\
\hline $\begin{array}{r}\text { Use } \\
\text { Associativity }\end{array}$ & Yes \\
\hline $\begin{array}{r}\text { Coordinate } \\
\text { Systems }\end{array}$ & No \\
\hline $\begin{array}{r}\text { Reader } \\
\text { Mode Saves } \\
\text { Updated File }\end{array}$ & No \\
\hline $\begin{array}{r}\text { Use } \\
\text { Instances }\end{array}$ & Yes \\
\hline $\begin{array}{r}\text { Smart CAD } \\
\text { Update }\end{array}$ & No \\
\hline $\begin{array}{r}\text { Compare } \\
\text { Parts On } \\
\text { Update }\end{array}$ & No \\
\hline $\begin{array}{r}\text { Attach File } \\
\text { Via Temp } \\
\text { File }\end{array}$ & Yes \\
\hline $\begin{array}{r}\text { Temporary } \\
\text { Directory }\end{array}$ & C:IUsersIJhon\AppDatalLocallTemp \\
\hline $\begin{array}{r}\text { Analysis } \\
\text { Type }\end{array}$ & 3-D \\
\hline $\begin{array}{r}\text { Decompose } \\
\text { Disjoint } \\
\text { Geometry }\end{array}$ & Yes \\
\hline $\begin{array}{r}\text { Enclosure } \\
\text { and } \\
\text { Symmetry } \\
\text { Processing }\end{array}$ & Yes \\
\hline
\end{tabular}

TABLE 3

Model (A4) > Geometry > Parts

\begin{tabular}{|r|c|}
\hline Object Name & Solid \\
\hline State & Meshed \\
\hline Graphics Properties \\
Visible & Yes \\
\hline Transparency & 1 \\
\hline Definition \\
\hline Stiffness Behavior & No \\
\hline Coordinate System & Dexible \\
\hline Reference & System \\
\hline Temperature & By Environment \\
\hline Material & \\
\hline Assignment & Structural Steel \\
\hline Nonlinear Effects & Yes \\
\hline Thermal Strain Effects & Yes \\
\hline
\end{tabular}

\begin{tabular}{|c|c|}
\hline \multicolumn{2}{|c|}{ Bounding Box } \\
\hline Length $\mathrm{X}$ & $42.017 \mathrm{~m}$ \\
\hline Length $\mathrm{Y}$ & $9.6403 \mathrm{~m}$ \\
\hline Length Z & 10. $\mathrm{m}$ \\
\hline \multicolumn{2}{|c|}{ Properties } \\
\hline Volume & $290.41 \mathrm{~m}^{3}$ \\
\hline Mass & $2.2798 \mathrm{e}+006 \mathrm{~kg}$ \\
\hline Centroid X & $1.0382 \mathrm{~m}$ \\
\hline Centroid $\mathrm{Y}$ & $0.87803 \mathrm{~m}$ \\
\hline Centroid Z & $0.35564 \mathrm{~m}$ \\
\hline Moment of Inertia Ip1 & $2.4241 \mathrm{e}+007 \mathrm{~kg} \cdot \mathrm{m}^{2}$ \\
\hline Moment of Inertia Ip2 & $2.4699 \mathrm{e}+008 \mathrm{~kg} \cdot \mathrm{m}^{2}$ \\
\hline Moment of Inertia Ip3 & $2.5508 \mathrm{e}+008 \mathrm{~kg} \cdot \mathrm{m}^{2}$ \\
\hline \multicolumn{2}{|c|}{ Statistics } \\
\hline Nodes & 48248 \\
\hline Elements & 26366 \\
\hline Mesh Metric & None \\
\hline \multicolumn{2}{|c|}{$\begin{array}{l}\text { Coordinate Systems } \\
\quad \text { TABLE } 4 \\
\text { A4) > Coordinate Systems > } \\
\text { Coordinate System }\end{array}$} \\
\hline
\end{tabular}

Object Name Global Coordinate System State Fully Defined Definition

Type Cartesian

Coordinate System ID $\quad 0$.

\begin{tabular}{l|l}
\multicolumn{2}{|c}{ Origin } \\
Origin $\mathrm{X}$ & $0 . \mathrm{m}$ \\
\hline Origin $\mathrm{Y}$ & $0 . \mathrm{m}$ \\
\hline Origin $\mathrm{Z}$ & $0 . \mathrm{m}$
\end{tabular}

\begin{tabular}{r|r} 
X Axis Data & [1.0.0.] \\
\hline Y Axis Data & {$[0.1 .0]$.} \\
\hline Z Axis Data & [0.0.1.] \\
\hline Mesh &
\end{tabular}

TABLE 5

Model (A4) $>$ Mesh Object Name Mesh

\begin{tabular}{|r|c|}
\hline Object Name & Mesh \\
\hline State & Solved \\
\hline Defaults & \\
\hline Physics Preference & Mechanical \\
\hline Relevance & 0 \\
Sizing & \\
\hline Use Advanced Size & Off \\
Function & Coarse \\
\hline Relevance Center & Default \\
\hline Element Size & \\
\hline
\end{tabular}


"Mircea cel Batran" Naval Academy Scientific Bulletin, Volume XX - 2017 - Issue 1

The journal is indexed in: PROQUEST / DOAJ / Crossref / EBSCOhost / INDEX COPERNICUS / DRJI / OAJI I JOURNAL INDEX I I2OR / SCIENCE LIBRARY INDEX / Google Scholar / Academic Keys/ ROAD Open Access I Academic Resources / Scientific Indexing Services / SCIPIO / JIFACTOR

\begin{tabular}{|c|c|}
\hline Initial Size Seed & Active Assembly \\
\hline Smoothing & Medium \\
\hline Transition & Fast \\
\hline Span Angle Center & Coarse \\
\hline Minimum Edge Length & $0.50 \mathrm{~m}$ \\
\hline \multicolumn{2}{|l|}{ Inflation } \\
\hline Use Automatic Inflation & None \\
\hline Inflation Option & Smooth Transition \\
\hline Transition Ratio & 0.272 \\
\hline Maximum Layers & 5 \\
\hline Growth Rate & 1.2 \\
\hline Inflation Algorithm & Pre \\
\hline View Advanced Options & No \\
\hline \multicolumn{2}{|c|}{ Patch Conforming Options } \\
\hline Triangle Surface Mesher & Program Controlled \\
\hline \multicolumn{2}{|c|}{ Patch Independent Options } \\
\hline Topology Checking & Yes \\
\hline \multicolumn{2}{|l|}{ Advanced } \\
\hline Shape Checking & $\begin{array}{l}\text { Standard } \\
\text { Mechanical }\end{array}$ \\
\hline Element Midside Nodes & Program Controlled \\
\hline Straight Sided Elements & No \\
\hline Number of Retries & Default (4) \\
\hline Extra Retries For Assembly & Yes \\
\hline Rigid Body Behavior & $\begin{array}{l}\text { Dimensionally } \\
\text { Reduced }\end{array}$ \\
\hline Mesh Morphing & Disabled \\
\hline \multicolumn{2}{|c|}{ Defeaturing } \\
\hline Pinch Tolerance & Please Define \\
\hline Generate Pinch on Refresh & No \\
\hline $\begin{array}{r}\text { Automatic Mesh Based } \\
\text { Defeaturing }\end{array}$ & On \\
\hline Defeaturing Tolerance & Default \\
\hline \multicolumn{2}{|l|}{ Statistics } \\
\hline Nodes & 48248 \\
\hline Elements & 26366 \\
\hline Mesh Metric & None \\
\hline \multicolumn{2}{|c|}{$\begin{array}{c}\text { TABLE } 6 \\
\text { Model }(\text { A4) }>\text { Mesh }>\text { Mesh Controls }\end{array}$} \\
\hline Object Name Face Sizi & \begin{tabular}{|l|l|} 
ing & Face Sizing 2 \\
\end{tabular} \\
\hline State & Ally Defined \\
\hline \multicolumn{2}{|l|}{ Scope } \\
\hline Scoping Method & Geometry Selection \\
\hline Geometry & 1 Face \\
\hline \multicolumn{2}{|l|}{ Definition } \\
\hline Suppressed & No \\
\hline
\end{tabular}

\begin{tabular}{|r|c|}
\hline Type & Element Size \\
\hline Element Size & $0.1 \mathrm{~m}$ \\
\hline Behavior & Soft \\
\hline
\end{tabular}

Static Structural (A5)

TABLE 7

Model (A4) > Analysis

Object Name Static Structural (A5)

State Solved

Definition

Physics Type Structural

Analysis Type Static Structural

Solver Target Mechanical APDL

Options

\begin{tabular}{|r|c|}
\hline Environment Temperature & $22 .{ }^{\circ} \mathrm{C}$ \\
\hline Generate Input Only & No \\
\hline
\end{tabular}

TABLE 8

Model (A4) > Static Structural (A5) $>$ Analysis Settings

\begin{tabular}{|c|c|}
\hline Object Name & Analysis Settings \\
\hline State & Fully Defined \\
\hline \multicolumn{2}{|c|}{ Step Controls } \\
\hline Number Of Steps & 1. \\
\hline $\begin{array}{r}\text { Current Step } \\
\text { Number }\end{array}$ & 1. \\
\hline Step End Time & 1. $\mathrm{s}$ \\
\hline Auto Time Stepping & Program Controlled \\
\hline \multicolumn{2}{|c|}{ Solver Controls } \\
\hline Solver Type & Program Controlled \\
\hline Weak Springs & Program Controlled \\
\hline Large Deflection & Off \\
\hline Inertia Relief & Off \\
\hline \multicolumn{2}{|c|}{ Restart Controls } \\
\hline $\begin{array}{r}\text { Generate Restart } \\
\text { Points }\end{array}$ & Program Controlled \\
\hline $\begin{array}{l}\text { Retain Files After } \\
\text { Full Solve }\end{array}$ & No \\
\hline \multicolumn{2}{|c|}{ Nonlinear Controls } \\
\hline $\begin{array}{r}\text { Newton-Raphson } \\
\text { Option }\end{array}$ & Program Controlled \\
\hline Force Convergence & Program Controlled \\
\hline $\begin{array}{r}\text { Moment } \\
\text { Convergence }\end{array}$ & Program Controlled \\
\hline $\begin{array}{l}\text { Displacement } \\
\text { Convergence }\end{array}$ & Program Controlled \\
\hline $\begin{array}{r}\text { Rotation } \\
\text { Convergence }\end{array}$ & Program Controlled \\
\hline Line Search & Program Controlled \\
\hline Stabilization & Off \\
\hline
\end{tabular}


"Mircea cel Batran" Naval Academy Scientific Bulletin, Volume XX - 2017 - Issue 1

The journal is indexed in: PROQUEST / DOAJ / Crossref / EBSCOhost / INDEX COPERNICUS / DRJI / OAJI I JOURNAL INDEX I I2OR / SCIENCE LIBRARY INDEX / Google Scholar / Academic Keys/ ROAD Open Access I Academic Resources / Scientific Indexing Services / SCIPIO / JIFACTOR

\begin{tabular}{|c|c|}
\hline \multicolumn{2}{|c|}{ Output Controls } \\
\hline Stress & Yes \\
\hline Strain & Yes \\
\hline Nodal Forces & No \\
\hline $\begin{array}{r}\text { Contact } \\
\text { Miscellaneous }\end{array}$ & No \\
\hline $\begin{array}{r}\text { General } \\
\text { Miscellaneous }\end{array}$ & No \\
\hline Store Results At & All Time Points \\
\hline \multicolumn{2}{|c|}{ Analysis Data Management } \\
\hline $\begin{array}{r}\text { Solver Files } \\
\text { Directory }\end{array}$ & $\mathrm{K}: \mid 21$ filesIdp0ISYSIMECH \\
\hline Future Analysis & None \\
\hline $\begin{array}{r}\text { Scratch Solver Files } \\
\text { Directory }\end{array}$ & \\
\hline Save MAPDL db & No \\
\hline $\begin{array}{r}\text { Delete Unneeded } \\
\text { Files }\end{array}$ & Yes \\
\hline Nonlinear Solution & No \\
\hline Solver Units & Active System \\
\hline Solver Unit System & mks \\
\hline
\end{tabular}

\section{TABLE 9}

Model (A4) > Static Structural (A5) > Loads

\begin{tabular}{|r|c|c|}
\hline Object Name & $\begin{array}{r}\text { Fixed } \\
\text { Support }\end{array}$ & Force \\
\hline State & \multicolumn{2}{|c|}{ Fully Defined } \\
\hline $\begin{array}{r}\text { Scoping } \\
\text { Method }\end{array}$ & \multicolumn{2}{|c|}{ Geometry Selection } \\
\hline Geometry & 3 Faces & 1 Face \\
\hline \multicolumn{3}{|c|}{ Definition } \\
\hline Type & $\begin{array}{c}\text { Fixed } \\
\text { Support }\end{array}$ \\
\hline Suppressed & \multicolumn{2}{|c|}{ Force } \\
\hline Define By & \multicolumn{2}{c|}{ Components } \\
\hline $\begin{array}{r}\text { Coordinate } \\
\text { System }\end{array}$ & & $\begin{array}{c}\text { Global Coordinate } \\
\text { System }\end{array}$ \\
\hline X Component & & 0. N (ramped) \\
\hline Y Component & & -2121. N (ramped) \\
\hline Z Component & & 0. N (ramped) \\
\hline
\end{tabular}

FIGURE 1

Model (A4) > Static Structural (A5) > Force

Solution (A6)

TABLE 10

Model (A4) > Static Structural (A5) > Solution

Object Name Solution (A6)

State Solved

\begin{tabular}{|r|c|}
\hline \multicolumn{2}{|c|}{ Adaptive Mesh Refinement } \\
\hline Max Refinement Loops & 1. \\
\hline Refinement Depth & 2. \\
\hline Information \\
\hline Status & Done \\
\hline TABLE 11
\end{tabular}

Model (A4) $>$ Static Structural $($ A5) $>$ Solution

(A6) $>$ Solution Information

\begin{tabular}{|r|c|}
\hline Object Name & $\begin{array}{c}\text { Solution } \\
\text { Information }\end{array}$ \\
\hline Solution Information & Solved \\
\hline Solution Output & Solver Output \\
\hline Newton-Raphson Residuals & 0 \\
\hline Update Interval & 2.5 s \\
\hline Display Points & All \\
\hline FE Connection Visibility \\
\hline Activate Visibility & Yes \\
\hline Display & All FE Connectors \\
\hline Draw Connections Attached & All Nodes \\
\hline To & \\
\hline Visible Color Results & Connection Type \\
\hline Line Thickness & No \\
\hline Display Type & Lingle \\
\hline TABLE 12 & \\
\hline
\end{tabular}

TABLE 12

Model (A4) $>$ Static Structural (A5) $>$ Solution (A6) $>$ Results

\begin{tabular}{|c|c|c|}
\hline Object Name & $\begin{array}{c}\text { Total } \\
\text { Deformation }\end{array}$ & $\begin{array}{c}\text { Equivalent } \\
\text { Elastic Strain }\end{array}$ \\
\hline State & \multicolumn{2}{|c|}{ Solved } \\
\hline \multicolumn{3}{|c|}{ Scope } \\
\hline Scoping Method & \multicolumn{2}{|c|}{ Geometry Selection } \\
\hline Geometry & \multicolumn{2}{|c|}{ All Bodies } \\
\hline \multicolumn{3}{|c|}{ Definition } \\
\hline Type & $\begin{array}{c}\text { Total } \\
\text { Deformation }\end{array}$ & $\begin{array}{c}\text { Equivalent } \\
\text { Elastic Strain }\end{array}$ \\
\hline By & \multicolumn{2}{|c|}{ Time } \\
\hline Display Time & \multicolumn{2}{|c|}{ Last } \\
\hline $\begin{array}{r}\text { Calculate Time } \\
\text { History }\end{array}$ & \multicolumn{2}{|c|}{ Yes } \\
\hline \multicolumn{3}{|l|}{ Identifier } \\
\hline Suppressed & \multicolumn{2}{|c|}{ No } \\
\hline \multicolumn{3}{|c|}{ Results } \\
\hline Minimum & $0 . \mathrm{m}$ & $\begin{array}{c}1.8291 \mathrm{e}-020 \\
\mathrm{~m} / \mathrm{m}\end{array}$ \\
\hline Maximum & $\begin{array}{c}4.8344 \mathrm{e}-009 \\
\mathrm{~m}\end{array}$ & $\begin{array}{c}2.8581 \mathrm{e}-009 \\
\mathrm{~m} / \mathrm{m}\end{array}$ \\
\hline
\end{tabular}


"Mircea cel Batran" Naval Academy Scientific Bulletin, Volume XX - 2017 - Issue 1 The journal is indexed in: PROQUEST / DOAJ / Crossref / EBSCOhost / INDEX COPERNICUS / DRJI / OAJI I JOURNAL INDEX I I2OR / SCIENCE LIBRARY INDEX / Google Scholar / Academic Keys/ ROAD Open Access I Academic Resources / Scientific Indexing Services / SCIPIO I JIFACTOR

\begin{tabular}{|r|c|c|}
\hline \multicolumn{3}{|c|}{ Minimum Value Over Time } \\
\hline Minimum & $0 . \mathrm{m}$ & $\begin{array}{c}1.8291 \mathrm{e}-020 \\
\mathrm{~m} / \mathrm{m}\end{array}$ \\
\hline Maximum & $0 . \mathrm{m}$ & $\begin{array}{c}1.8291 \mathrm{e}-020 \\
\mathrm{~m} / \mathrm{m}\end{array}$ \\
\hline Maximum Value Over Time
\end{tabular}

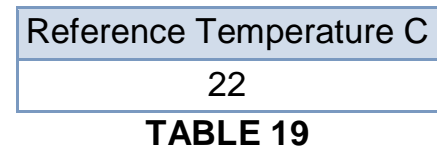

Structural Steel > Alternating Stress Mean Stress

\begin{tabular}{|c|c|c|}
\hline Alternating Stress $\mathrm{Pa}$ & Cycles & Mean Stress Pa \\
\hline $3.999 \mathrm{e}+009$ & 10 & 0 \\
\hline $2.827 \mathrm{e}+009$ & 20 & 0 \\
\hline $1.896 \mathrm{e}+009$ & 50 & 0 \\
\hline $1.413 \mathrm{e}+009$ & 100 & 0 \\
\hline $1.069 \mathrm{e}+009$ & 200 & 0 \\
\hline $4.41 \mathrm{e}+008$ & 2000 & 0 \\
\hline $2.62 \mathrm{e}+008$ & 10000 & 0 \\
\hline $2.14 \mathrm{e}+008$ & 20000 & 0 \\
\hline $1.38 \mathrm{e}+008$ & $1 . \mathrm{e}+005$ & 0 \\
\hline $1.14 \mathrm{e}+008$ & $2 . \mathrm{e}+005$ & 0 \\
\hline $8.62 \mathrm{e}+007$ & $1 . \mathrm{e}+006$ & 0 \\
\hline
\end{tabular}

TABLE 20

Structural Steel > Strain-Life Parameters

\begin{tabular}{|c|c|c|c|c|c|}
\hline $\begin{array}{r}\text { Strengt } \\
\mathrm{h} \\
\text { Coeffici } \\
\text { ent Pa }\end{array}$ & $\begin{array}{r}\text { Streng } \\
\text { th } \\
\text { Expon } \\
\text { ent }\end{array}$ & $\begin{array}{r}\text { Ductilit } \\
y \\
\text { Coeffici } \\
\text { ent }\end{array}$ & $\begin{array}{r}\text { Ductilit } \\
y \\
\text { Expon } \\
\text { ent }\end{array}$ & $\begin{array}{r}\text { Cyclic } \\
\text { Strengt } \\
\mathrm{h} \\
\text { Coeffici } \\
\text { ent } \mathrm{Pa}\end{array}$ & $\begin{array}{r}\text { Cyclic } \\
\text { Strain } \\
\text { Harden } \\
\text { ing } \\
\text { Expon } \\
\text { ent }\end{array}$ \\
\hline $\begin{array}{c}9.2 \mathrm{e}+0 \\
08\end{array}$ & -0.106 & 0.213 & -0.47 & $\begin{array}{c}1 . e+00 \\
9\end{array}$ & 0.2 \\
\hline
\end{tabular}

TABLE 21

Structural Steel > Isotropic Elasticity

\begin{tabular}{|c|c|c|c|c|}
\hline $\begin{array}{r}\text { Temperat } \\
\text { ure C }\end{array}$ & $\begin{array}{r}\text { Young } \\
\text { 's } \\
\text { Modul } \\
\text { us Pa }\end{array}$ & $\begin{array}{r}\text { Poisso } \\
\text { n's } \\
\text { Ratio }\end{array}$ & $\begin{array}{r}\text { Bulk } \\
\text { Modulus } \\
\mathrm{Pa}\end{array}$ & $\begin{array}{r}\text { Shear } \\
\text { Modulus } \\
\mathrm{Pa}\end{array}$ \\
\hline & $\begin{array}{c}2 . e+0 \\
11\end{array}$ & 0.3 & $\begin{array}{c}1.6667 \mathrm{e}+ \\
011\end{array}$ & $\begin{array}{c}7.6923 e+ \\
010\end{array}$ \\
\hline
\end{tabular}

TABLE 22

Structural Steel > Isotropic Relative Permeability Relative Permeability

Structural Steel > Tensile Yield Strength

\begin{tabular}{|c|}
\hline Tensile Yield Strength $\mathrm{Pa}$ \\
\hline $2.5 \mathrm{e}+008$ \\
\hline TABLE 17
\end{tabular}

Structural Steel > Tensile Ultimate Strength

\begin{tabular}{|c|}
\hline Tensile Ultimate Strength $\mathrm{Pa}$ \\
\hline $4.6 \mathrm{e}+008$ \\
\hline TABLE 18
\end{tabular}

Structural Steel > Isotropic Secant Coefficient of Thermal Expansion 
"Mircea cel Batran" Naval Academy Scientific Bulletin, Volume XX - 2017 - Issue 1

The journal is indexed in: PROQUEST / DOAJ / Crossref / EBSCOhost / INDEX COPERNICUS / DRJI / OAJI I JOURNAL INDEX I I2OR / SCIENCE LIBRARY INDEX / Google Scholar / Academic Keys/ ROAD Open Access I Academic Resources / Scientific Indexing Services / SCIPIO / JIFACTOR

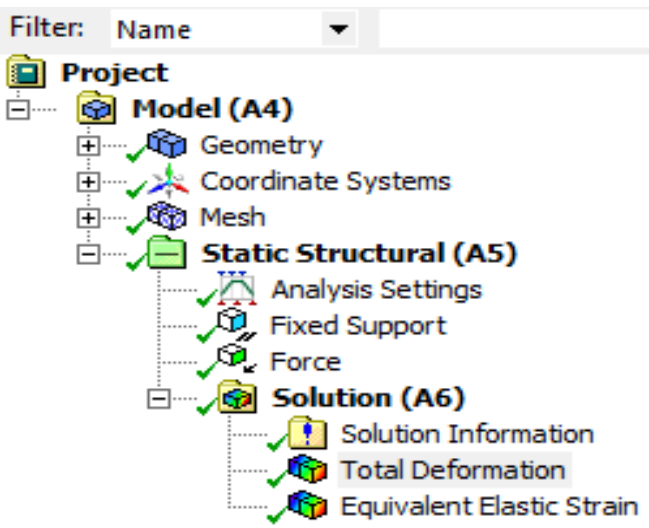

Settings within Ansys Workbench Program Outline are made and the steps required to achieve the Ansys Workbench correct simulation. You can manage the taught elements considered for analysis and geometry, how it will achieve meshing and managing various requests that appear on track in the study.

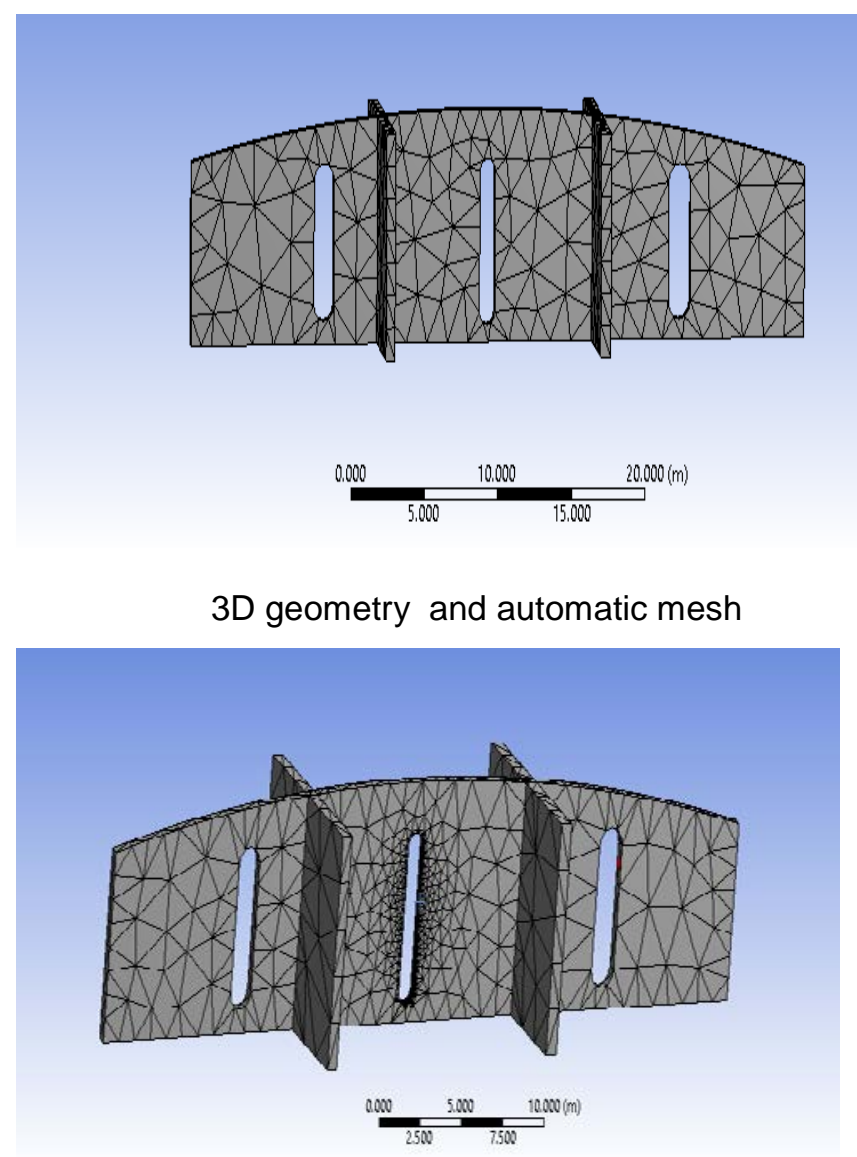

3D geometry transverse element of the vessel. Meshing controlled situation with refining zone 1

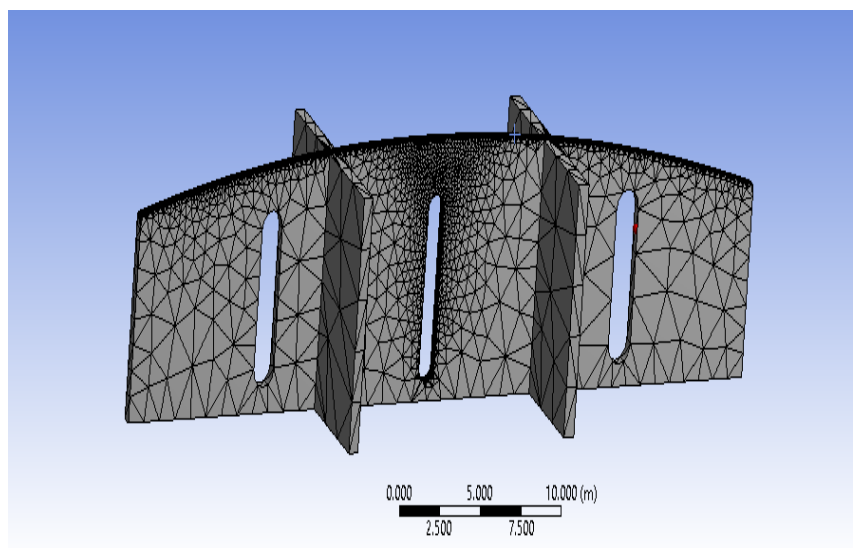

Controlled meshing situation with refining zone 2

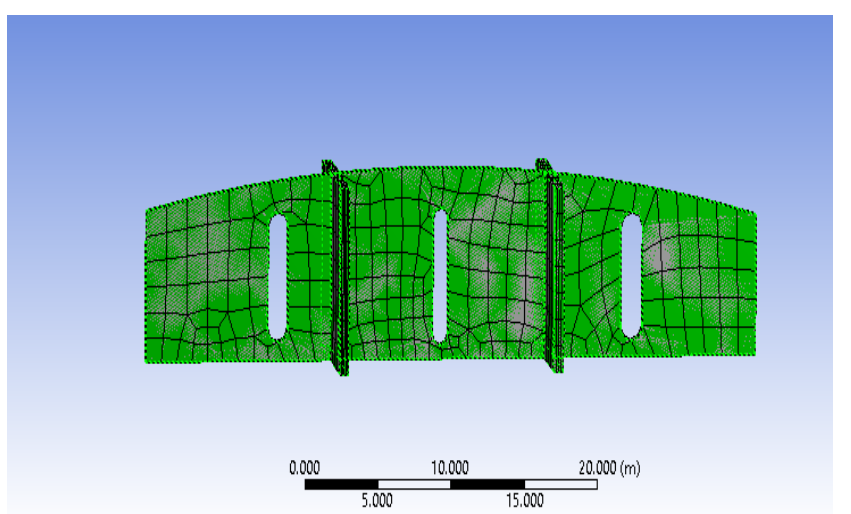

Presentation working method

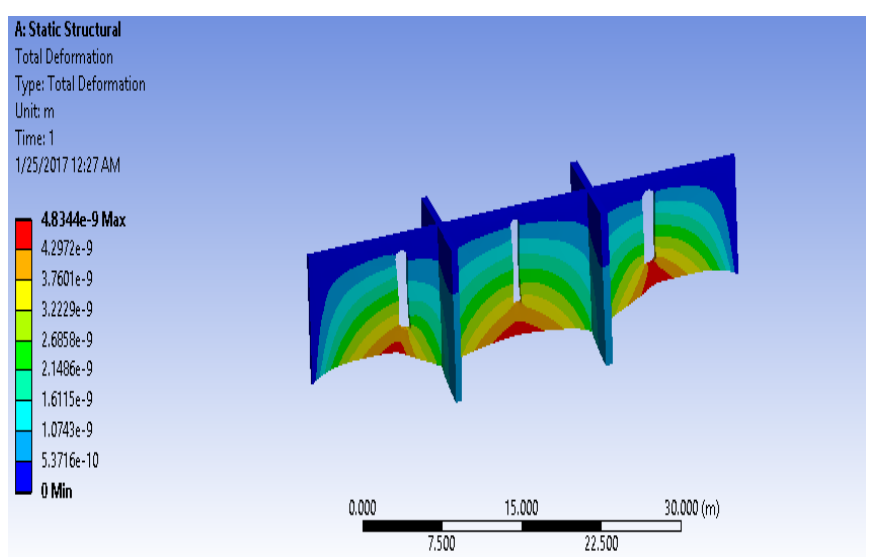

Results 3D to the ship structure (deformations 1 ) 
"Mircea cel Batran" Naval Academy Scientific Bulletin, Volume XX - 2017 - Issue 1 The journal is indexed in: PROQUEST / DOAJ / Crossref / EBSCOhost / INDEX COPERNICUS / DRJI / OAJI I JOURNAL INDEX I I2OR / SCIENCE LIBRARY INDEX / Google Scholar / Academic Keys/ ROAD Open Access I Academic Resources / Scientific Indexing Services / SCIPIO / JIFACTOR

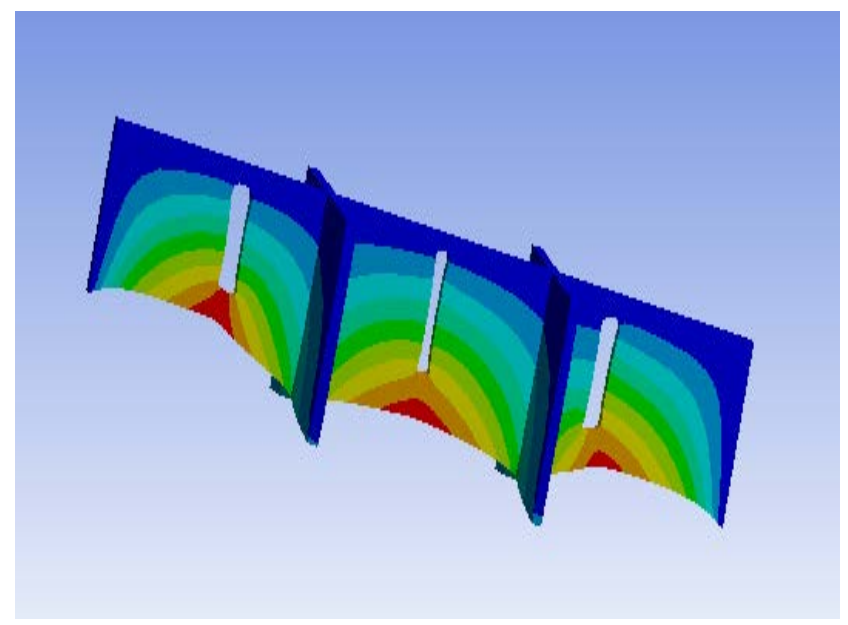

Results 3D to the ship structure (deformations 2)

\section{Conclusion}

In this study, Ansys statical loads method is used for an actual simulation case study based on a transverse ship beam. Due to impossibility of experimental tests, the presented beam model was analised in Ansys numerical simulation. All result presented as beam deformation and stress for different mesh use. The Ansys simulation presented for ship beam is a fast way to solve static load analysis for any ship element.

\section{Bibliography}

[1] Ilie Patrichi, Ali Beazit, Iordan Novac, The strenght structure and the stress of shell plating made of fibreglass reinforced plastics FRP, Analele Universităţii Maritime din Constanţa, anul VI, vol. 8, 2005, pag. 265 - 274, ISSN 1582-3601.

[2] Călimănescu I., Stan L. C., Computer fluid dynamics (CFD)study of a micro annular gear pump, Atom 2016, Conference Paper.

[3] Stan L. Călimănescu I.,. C.A New innovative turbocharger concept numerically tested and optimised with cfd, 2016, Conference Paper.

$[4]{ }^{\star \star *}$ Ansys software documents and help 